\title{
Joint estimation of water column and sediment acoustic properties from broadband towed array data using modal inverse method
}

\author{
Subramaniam D. Rajan ${ }^{\text {a) }}$ \\ Scientific Solutions, Incorporated, 99 Perimeter Road, Nashua, New Hampshire 03063 \\ G. V. Anand ${ }^{\text {b) }}$ and P. V. Nagesh \\ Indian Institute of Science, Bangalore, India, 560012
}

(Received 28 March 2006; revised 7 June 2006; accepted 18 June 2006)

\begin{abstract}
Broadband data, collected on a towed horizontal array of length $2375 \mathrm{~m}$ during a geophysical survey, are used to perform joint inversion for the water column and sediment acoustic parameters using modal inverse technique [S. D. Rajan, J. F. Lynch, and G. V. Frisk, J. Acoust. Soc. Am. 82, 998-1017 (1987)]. To perform the inversion based on modal wave numbers, we first obtain the pressure field as a function of range at a discrete set of frequencies from the broadband data. The sources of errors and their impact on the estimation of modal wave number from such data are discussed. Due to the possibility of large errors in the estimation of the modal wave numbers of the higher-order modes, the inversion was performed using the modal wave numbers of the dominant lower-order modes. The resolution estimates indicate that the wave speeds in the water column and top three sediment layers have resolution lengths that are approximately 1.5 times the layer thicknesses. With respect to deeper sediment layers, the resolution lengths are large, as only lower-order modes were used in inversion. These results are consistent with a posteriori variance estimates. The model from modal inverse can serve as the initial model for nonlinear methods, such as those based on matched field processing [P. V. Nagesh, S. D. Rajan, and G. V. Anand, Conference on Underwater Acoustics Measurements: Technologies and Results, Heraklion, Crete, Greece (2005)]. ( 2006 Acoustical Society of America. [DOI: 10.1121/1.2221558]
\end{abstract}

PACS number(s): 43.30.Pc [AIT]

Pages: $1324-1334$

\section{INTRODUCTION}

A method for estimating sediment compressional wave speed profiles from the wave numbers of propagating modes in a shallow water waveguide has been reported in the literature. ${ }^{1}$ The applicability of this approach has been demonstrated in a number of field experiments. ${ }^{3,4}$ In these experiments, the data were collected on a synthetic aperture horizontal array using a harmonic point source. In the analysis presented here, we use broadband data collected on a towed horizontal array by a survey agency conducting a geophysical survey in for an oil company. Due to security and other considerations, the location of the experiment and the sound speed profile in the water column during the experiment was not provided to us. An approximate estimate of the water column depth was, however, provided. We, therefore, investigate joint inversion for water column and sediment compressional wave speeds and their thicknesses by modal inverse method. Even though the theoretical framework for modal inverse has been presented in earlier papers, ${ }^{1,3,4}$ its application to broadband data collected on a horizontal array has not yet been studied.

The goal of this paper is to examine the feasibility of using data that are routinely collected by oil companies for estimating the geoacoustic properties of near-surface sedi-

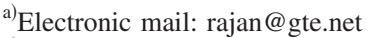

${ }^{b)}$ Electronic mail: anandgv@ece.iisc.ernet.in
}

ment layers in a shallow water environment. While the analysis conducted by the seismic industry is directed toward defining the layering structure of the sediments (i.e., locating the reflectors/interfaces), we seek a description of the sediment in terms of the wave speeds in the layers and their thicknesses. The horizontal array used in the survey was approximately $2.4 \mathrm{~km}$ long. The array had 96 hydrophones with an interelement distance of $25 \mathrm{~m}$, and was towed at a depth of $6 \mathrm{~m}$. The source, an air gun array, was hung from the ship that was towing the array. The center of the source array was at a depth of $6 \mathrm{~m}$. The distance from the source to the closest receiver on the hydrophone array was $200 \mathrm{~m}$. The approximate depth of the water column, as indicated by the survey agency, was $35 \mathrm{~m}$. During the experiment, some of the receivers were blanked out and no data were collected at these receivers. The sampling rate was $500 \mathrm{~Hz}$, and each channel recorded $3 \mathrm{~s}$ of data after each transmission. The data acquisition system was designed to trigger the transmitter and the receivers simultaneously. The spectrum of the signal received by the hydrophones on the array indicated that the signal had considerable energy in the frequency interval from 15 to $50 \mathrm{~Hz}$. In this paper, we use the broadband data to first obtain the modal wave numbers at a set of frequencies, and then use these data to jointly estimate the water column properties and sediment compressional wave speed profiles. We consider data acquired from three shots (Shot 5, Shot 50, and Shot 95). 
The signal at each receiver is a time series $p\left(t, r_{n}\right)$ where $p$ is the pressure field, $t$ is the time, and $r_{n}$ is the range of the $n$th receiver on the horizontal array. By performing the discrete Fourier transform of the received signal with respect to $t$, we obtain the pressure field $p\left(\omega, r_{n}\right)$ as a function of range at a discrete set of frequencies. The data, thus transformed into the frequency domain, are used to estimate the water column and sediment acoustic properties.

It can be shown that in a range independent circular symmetric ocean, the pressure field $p(r, \omega)$ at a range $r$, due to a harmonic point source of radian frequency $\omega$ and the wave number spectrum $G\left(k_{r}, \omega\right)$ at the same frequency, are related by the equation ${ }^{5}$

$$
G\left(k_{r}, \omega\right)=\int_{0}^{\infty} p(r, \omega) J_{0}\left(k_{r} r\right) r d r,
$$

where $k_{r}$ is the horizontal wave number and $J_{0}$ is the Bessel function of zero order. The inverse of this relation, as shown below, yields the pressure field from the wave number spectrum

$$
p(r, \omega)=\int_{0}^{\infty} G\left(k_{r}, \omega\right) J_{0}\left(k_{r} r\right) k_{r} d k_{r} .
$$

In the far field, the argument $k_{r} r$ has large values, and we can use the asymptotic approximation

$$
J_{0}\left(k_{r} r\right) \approx \frac{\exp \left[i\left(k_{r} r-\pi / 4\right)\right]}{\sqrt{2 \pi k_{r} r}}, \quad k_{r} r \gg 1,
$$

for the Bessel function. Insertion of this approximation in Eq. (1) yields the following relationship for the wave number spectrum.

$$
G\left(k_{r}, \omega\right)=\frac{\exp (-i \pi / 4)}{\sqrt{2 \pi k_{r}}} \int_{0}^{\infty} \sqrt{r} p(r, \omega) \exp \left(-i k_{r} r\right) d r .
$$

For any given $\omega$, the wave number spectrum $G\left(k_{r}, \omega\right)$ has peaks whose locations on the wave number axis are the modal wave numbers of the propagating modes at that $\omega$. The modal wave numbers of the propagating modes in a shallow water waveguide are dependent on the acoustic and geometric parameters of the shallow water environment. Rajan et al. ${ }^{1}$ describes a scheme wherein modal wave numbers obtained from a field experiment can be used to estimate the sediment acoustic properties. We adopt this procedure to estimate the water column and sediment acoustic properties here.

\section{ESTIMATION OF MODAL WAVE NUMBERS}

The data were collected on a horizontal array towed by a ship carrying the source. This experimental setup was not designed to collect data readily adaptable for estimating the wave number spectrum at various frequencies. As indicated in Sec. I, the acquired data are transformed to obtain the pressure field as a function of range at a given frequency. In using these data to obtain the modal wave numbers, the following issues need to be addressed:
1. Aliasing due to inadequate spatial sampling of the pressure field,

2. Errors in the estimation of modal wave numbers due to errors in range and source/receiver depths,

3. Doppler effect due to array motion,

4. Estimation errors due to the short aperture of data, and

5. Noise in the broadband data.

\section{A. Inadequate sampling of the field}

The wave number spectrum at a given frequency can be estimated by using a discretized version of Eq. (4). In order to avoid aliasing, the acoustic field has to be sampled according to the Nyquist criterion, i.e., at intervals of $\lambda / 2$ or less, where $\lambda$ is the lowest wavelength of interest. A much denser sampling $(\lambda / 4)$ is normally adopted. Hence, at a frequency of $25 \mathrm{~Hz}$ and a minimum compressional wave speed of $1500 \mathrm{~m} / \mathrm{s}$ in the region of interest, the field has to be sampled at intervals of $15 \mathrm{~m}$. The data acquired on the horizontal array have a sampling interval of $25 \mathrm{~m}$, with some of the receivers blanked out.

We now demonstrate that the undersampled field data can be interpolated to give the required sampling rate, without significant error in amplitude and phase, using simulated data similar to the field data. In order to generate the simulated data, we consider a horizontal array with 100 receivers with an interelement spacing of $25 \mathrm{~m}$. A source is placed in the end-fire position at a distance of $250 \mathrm{~m}$ from the first receiver on the horizontal array. This configuration simulates the setup used in the collection of the field data that is the subject of study in this paper. The source signal is assumed to be a Gaussian pulse with a central frequency of $35 \mathrm{~Hz}$ and a band width of $50 \mathrm{~Hz}$. The signal received at each of the 100 receivers is obtained using the pulse propagation module in OASES. ${ }^{6}$ The shallow water environment used in computing the simulated data was representative of the environment where the field data were collected. Further, we assumed that the properties of the medium are range independent. The simulated pressure field, $p_{\text {sim }}\left(t, r_{n}\right)$, is a time series and represents the pressure field at the $n$th receiver at range $r_{n}$. We process these data, as described in Sec. I, and obtain the pressure field $p_{\text {sim }}\left(\omega, r_{n}\right)$ as a function of frequency and range.

As the receiver spacing is $25 \mathrm{~m}$, we have to sample the pressure field on a much finer grid in order to obtain the wave number spectrum over a range of frequencies. The field $p_{\text {sim }}\left(\omega, r_{n}\right)$ is interpolated to give $p_{\text {sim }}\left(\omega, r_{\text {int }}\right)$, where $r_{\text {int }}$ is a set of ranges at a much higher sampling rate so as to avoid aliasing. The interpolation is performed using the cubic spline routine. Since the phase rapidly changes, we slowed down the phase by multiplying the field by the term $\exp \left(i k r_{n}\right)$ before performing the interpolation. After performing the interpolation, the correct phase was restored by multiplying the interpolated field by the term $\exp \left(-i k r_{\text {int }}\right)$. To check whether this scheme introduces error in the amplitude and phase of the interpolated field, we compute the pressure field $p_{\text {test }}\left(\omega, r_{\text {int }}\right)$ using the procedure used to obtain $p_{\text {sim }}\left(\omega, r_{n}\right)$, except that we now use a horizontal array where the element spacing corresponds to $r_{\text {int }}$. The magnitude and 

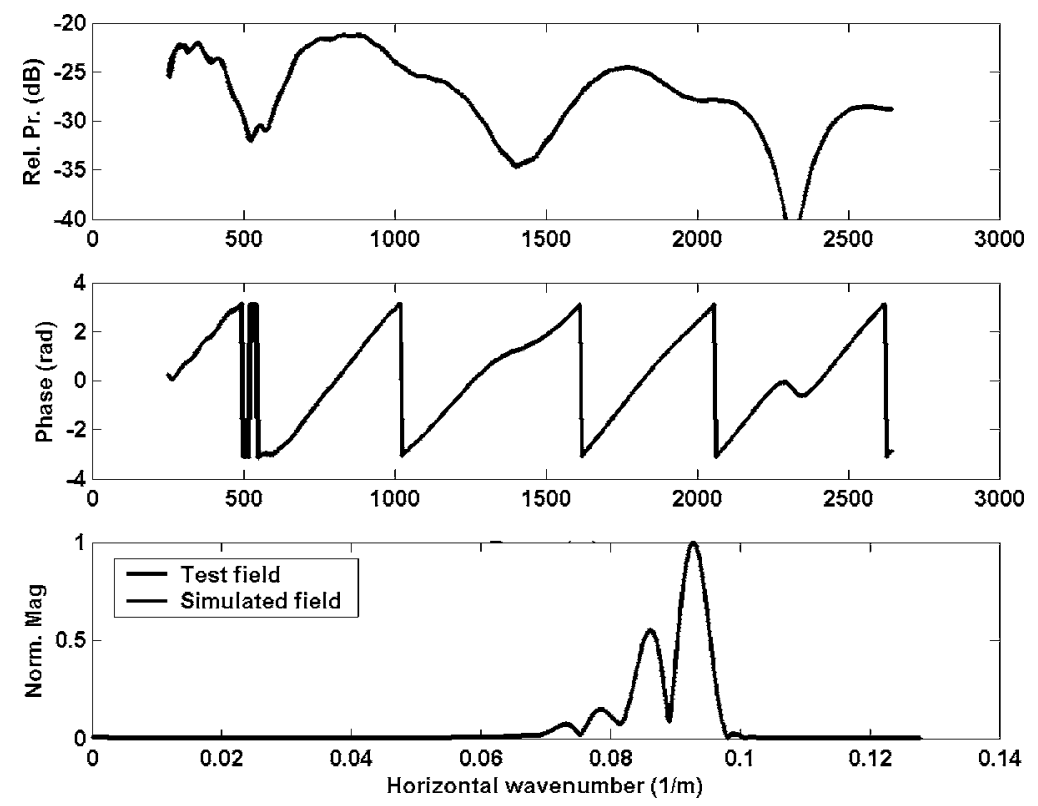

FIG. 1. The top panel shows the comparison between the magnitude of the field obtained with an interelement spacing of $6 \mathrm{~m}$ (dots) and the field computed with an interelement spacing of $25 \mathrm{~m}$, and interpolated to a grid spacing of $6 \mathrm{~m}$ (firm lines). The source frequency was $25 \mathrm{~Hz}$. The middle panel shows the comparison of the slowed down phase in the two cases. The results from the two cases are identical and, hence, only one line is seen in the plots. The bottom panel shows the wave number spectrum for the two cases, and here again the agreement is excellent. phase of the pressure field $p_{\text {sim }}\left(\omega, r_{\text {int }}\right)$ are compared with the amplitude and phase of $p_{\text {test }}\left(\omega, r_{\text {int }}\right)$ in Fig. 1 . These fields were computed at a frequency of $25 \mathrm{~Hz}$. We note that the agreements between the two fields are excellent. A comparison between the wave number spectra obtained by the two methods is also shown in Fig. 1. Similar interpolations performed on an acoustic field at higher frequencies (up to $100 \mathrm{~Hz}$ ) also showed negligible error arising from interpolation. These simulations show that broadband data, obtained at a set of receivers in a horizontal array can, by suitable processing, be converted into the field due to a harmonic source on a horizontal array, with adequate spatial sampling, in order to obtain the wave number spectrum.

\section{B. Impact of errors in receiver range and depth}

We have assumed that the horizontal array is perfectly straight with no distortion. Any distortion of the array will result in errors in the receiver position with respect to its range and depth. In this section, we investigate the effect of errors in receiver positions on the estimation of modal wave numbers. Since we assume circular symmetry for the ocean, lateral displacement of the array is ignored.

Let there be small random errors $\Delta r$ and $\Delta z$ in the range and depth of the receivers, whose nominal (assumed) range and depth are $r$ and $z_{r}$, respectively. The estimate of the wave number spectrum, obtained from acoustic measurements made on the perturbed array, is given by

$$
\begin{aligned}
\hat{G}\left(k_{r}, \omega\right)= & \frac{\exp (-i \pi / 4)}{\sqrt{2 \pi k_{r}}} \int_{r_{0}}^{R+r_{0}} \sqrt{r p}\left(r+\Delta r, z_{r}+\Delta z ; \omega\right) \\
& \times \exp \left(-i k_{r} r\right) d r,
\end{aligned}
$$

where $R$ is the aperture of the horizontal array and $r_{0}$ is the distance between the source and the nearest receiver on the array. The acoustic pressure $p\left(r, z_{s}, z_{r}\right)$ for a given source and receiver location is the sum of contributions from the set of propagating modes and is given by the expression ${ }^{7}$

$$
p\left(r, z_{s}, z_{r}\right)=\frac{(1+i)}{4 \sqrt{\pi}} \sum_{n=1}^{N} \frac{1}{\sqrt{k_{n} r}} \phi_{n}\left(z_{s}\right) \phi_{n}\left(z_{r}\right) \exp \left(i k_{n} r\right),
$$

where $N$ is the total number of propagating modes, $k_{n}$ is the wave number of the $n$th mode, $\phi_{n}\left(z_{s}\right)$ and $\phi_{n}\left(z_{r}\right)$ are the values of the $n$th mode function at the source and receiver depths, and $r$ is the range to the receiver from the source. Hence, the estimate of the wave number spectrum is given by the expression

$$
\begin{aligned}
\hat{G}\left(k_{r}\right)= & \frac{(1+i)}{4 \sqrt{\pi}} \sum_{n=1}^{N} \phi_{n}\left(z_{s}\right) \phi_{n}\left(z_{r}+\Delta z\right) \int_{r_{0}}^{r_{0}+R} \frac{1}{\sqrt{k_{n} k_{r}}} \\
& \times \exp \left[i k_{n}(r+\Delta r)\right] \exp \left(-i k_{r} r\right) d r .
\end{aligned}
$$

Assuming that the perturbation $\Delta r$ is a Gaussian random variable with zero mean and variance $\sigma^{2}$, we have $E\left[\exp \left(i k_{n} \Delta r\right)\right]=\exp \left(-k_{n}^{2} \sigma^{2} / 2\right)$, where $E[$.] denotes the expectation or statistical average. If the depth perturbations $\Delta z$ is small with zero mean, we have, ignoring the higherorder terms,

$$
E\left[\phi_{n}\left(z_{r}+\Delta z\right)\right] \approx E\left[\phi_{n}\left(z_{r}\right)+\Delta z \phi_{n}^{\prime}\left(z_{r}\right)\right]=\phi_{n}\left(z_{r}\right) .
$$

Hence, assuming that the range perturbation $\Delta r$ and the depth perturbation $\Delta z$ are statistically independent, we have

$$
\begin{aligned}
E\left[\hat{G}\left(k_{r}\right)\right]= & \frac{(1+i)}{4 \sqrt{\pi}} \sum_{n=1}^{N} \frac{\exp \left(-k_{n}^{2} \sigma^{2} / 2\right)}{\sqrt{k_{n} k_{r}}} \phi_{n}\left(z_{s}\right) \phi_{n}\left(z_{r}\right) \\
& \times \int_{r_{0}}^{r_{0}+R} \exp \left[i\left(k_{n}-k_{r}\right) r\right] d r .
\end{aligned}
$$

Therefore, small perturbations of the receiver range and depth result in a reduction in the estimated amplitude of the averaged wave number spectrum, and do not alter the location of the peaks, i.e., the modal wave numbers remain unchanged. While this is true for the averaged wave number spectrum, where the average is done over a large number of realizations, the field data have a single realization and there- 

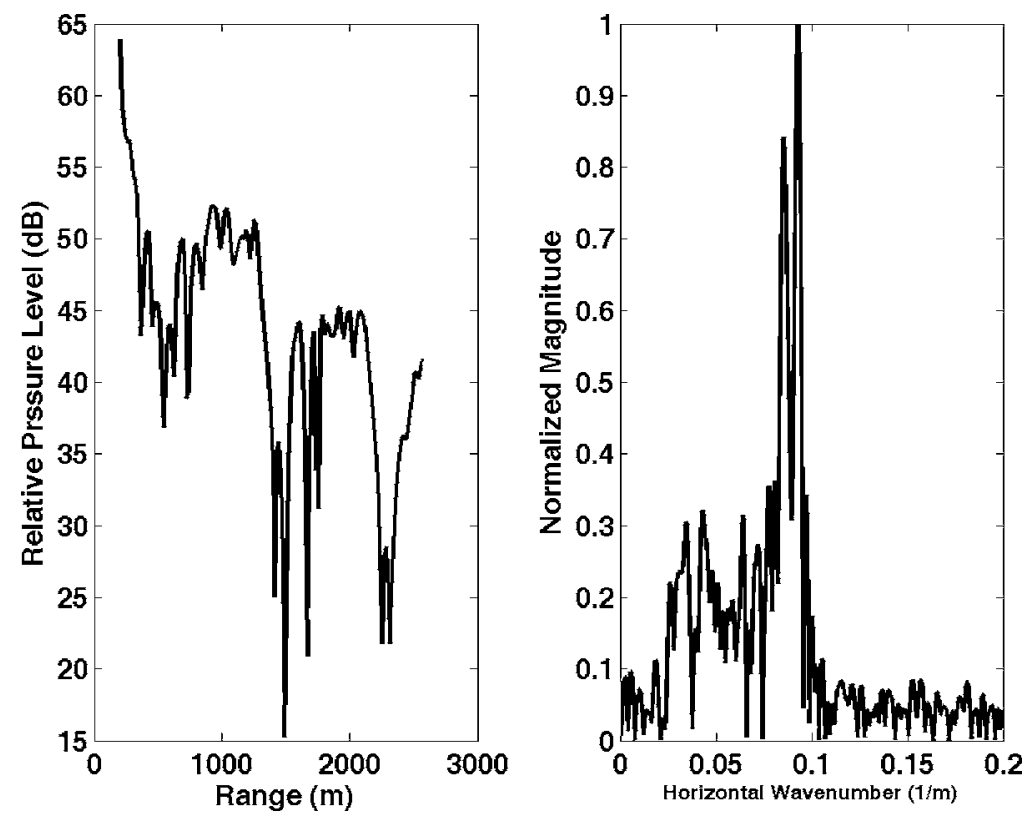

FIG. 2. The left panel shows the field at $25 \mathrm{~Hz}$ determined from Shot 50 data. The right panel shows the wave number spectrum. The wave number spectrum shows considerable energy beyond the water wave number. This results in the oscillatory structure seen in the pressure field. See the similarity between this and the plots in Fig. 3.

fore have phase errors that cause errors in the estimated modal wave numbers. Simulations indicate that the effect of an array perturbation on the estimation of modal wave numbers is negligible in the case of dominant lower-order modes at the frequencies of interest. Even in the case of weak higher-order modes, the errors in the estimation of modal wave numbers are small.

\section{Errors due to source and receiver motion}

In determining the wave numbers of the propagating modes, two sources of errors due to source/receiver motion have to be taken into account. The ship that tows the horizontal array is moving at an approximately constant speed. The source is suspended from the same ship that tows the receiver array. At the instant of source activation, we can consider both the source and the receivers to be stationary. Subsequently as the ship moves it does not affect the signal already transmitted, and hence we need to consider only the effect of receiver motion. Due to the receiver motion, the range to the receiver - at the time the signal arrives at the receiver-will be less than the nominal range based on the number of elements in the array and the interelement distance. The distance of each receiver, at the time the signal arrives at that receiver, is approximately the range at zero time (the time of shot activation) minus the distance traveled by the receiver, which is the ship speed multiplied by the time taken by the signal to reach the receiver.

The second correction to be applied is the Doppler correction, which is the impact of the receiver motion on the horizontal wave number. The corrected wave number is given by the expression $k_{n}^{*}=k_{n}\left[1+\left(v_{r}\right) / v_{g n}\right]$, 8,9 where $k_{n}^{*}$ is the corrected wave number for the $n$th mode, $v_{r}$ is the receiver speed, $v_{g n}$ is the group velocity of the $n$th mode, and $k_{n}$ is the wave number for a stationary receiver. The wave number values obtained from the experiment are to be corrected using the above expression in order to obtain the values corresponding to a stationary array. The frequency that the receiver senses will also be shifted due to its motion, and the shifted frequency $\omega^{*}$ is given by the approximate expression $\omega^{*} \approx \omega\left[1+v_{r} / c_{w}\right]$, where $c_{w}$ is the sound speed in the water column. An exact expression for the frequency shift is found in Ref. 9.

\section{Processing short aperture data}

If the wave number spectrum is obtained using Eq. (1), it will not be possible to resolve modes whose separation in the wave number space is less than $2 \pi / L$, where $L$ is the aperture of the array. The number of propagating modes and their wave numbers depend on the frequency of the acoustic source, the acoustic properties of the water column and sediment layers, and the layer thicknesses. Since we are seeking to determine these very acoustic properties of the shallow water environment, which are unknown, we examined the structure of the wave numbers over a range of frequencies $(15 \mathrm{~Hz}-50 \mathrm{~Hz})$ using the ocean model used to generate the simulated data. As we had indicated, the ocean model used to generate the simulated data is representative of the environment where the field data were collected. The lower-order modes in these cases were well separated and resolvable. The higher-order modes were however irresolvable. In the event that some of the modes are not well resolved, high-resolution methods that use schemes, such as the autoregressive spectral estimation method, ${ }^{10}$ MUSIC, ${ }^{11}$ and ESPRIT, ${ }^{11}$ can be employed.

\section{E. Noise in broadband data}

Finally, we study the effect of noise in the data. For this purpose, we use the data simulated as described in Sec. II A and add a small Gaussian distributed noise to the signal at each receiver. The noise is assumed to be uncorrelated between the receivers. The amount of noise added was such as to mimic the behavior observed in the field data, namely the oscillatory nature of the magnitude of the field - as shown in Fig. 2. In Fig. 3, the effect of noise in the data is shown. The left panel shows the pressure field for noise-free and noisy 

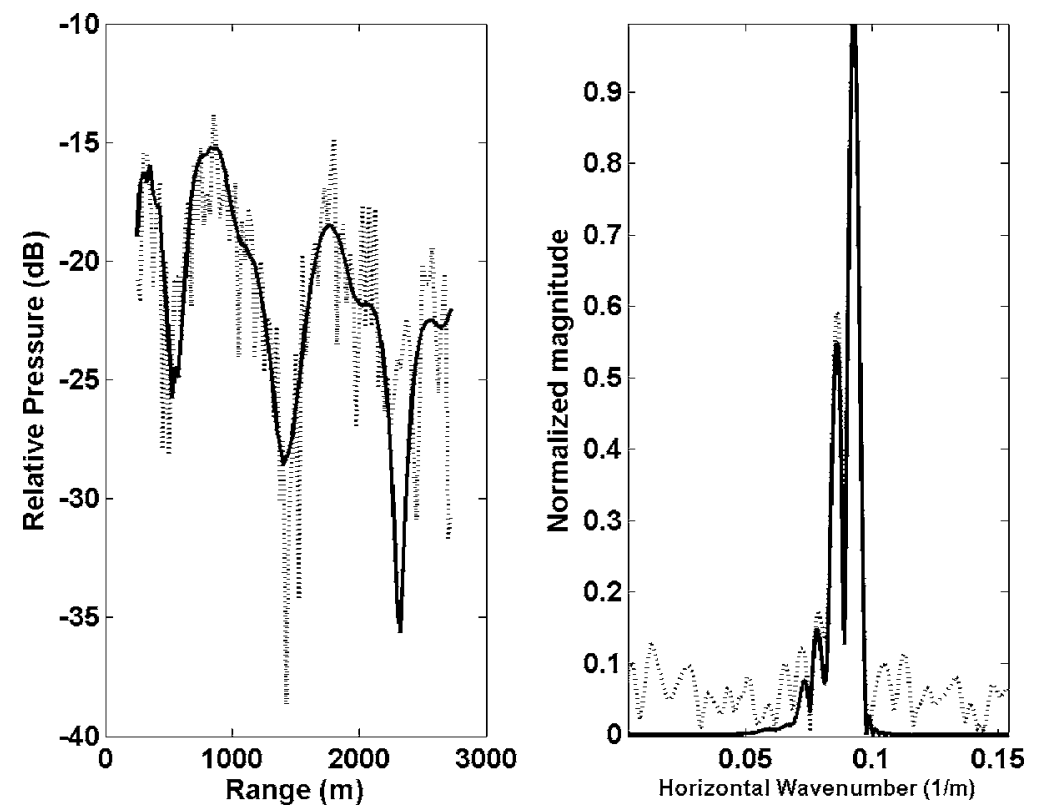

FIG. 3. The left panel indicates the field when the horizontal array data are corrupted by noise. This is compared with the field from the noise-free case. The right panel shows the wave number spectra for the two cases.

simulated data. On the right panel, we compare the wave number spectrum obtained with the noisy data with the spectrum for the noise-free data. We note that the lower-order modes, which are strong, can be easily detected in the wave number spectrum obtained from the noisy data. However, the higher-order modes have errors in their magnitude and location. Further, the wave number spectrum is also noisy. These simulations show the difficulty in estimating the wave numbers of the weak higher-order modes when noise is present in the data.

\section{INVERSION METHOD}

We now briefly describe the method for estimating the water column and sediment properties from modal wave numbers. This approach has been described extensively in the literature. ${ }^{1,3,4}$ The wave numbers of the propagating modes in the waveguide are obtained from the wave number spectrum. Let $k_{n}$ be the wave number of the $n$th mode, as obtained from the field measurement of the pressure field. Let us assume that the unknown quantities are the compressional wave speed in the water column and the sediment layers. All other parameters of the sediment layers and the water column are assumed known. We now assume that the compressional wave speed profile, representing the water column and the sediment layers, is given by $C_{b}(z)$. This initial estimate is normally obtained from archival data or other sources of information. Let this initial estimate be related to the true, but unknown, compressional wave speed profiles by the equation $C(z)=C_{b}(z)+\Delta C(z)$, where $\Delta C(z)$ is a small quantity which is the difference between the initial estimate of the compressional wave speed profile and the true profile. By solving the forward problem, we determine modal wave numbers for the initial ocean model. Let these be $k_{b n}$. In a range independent ocean environment, the difference, $\Delta k_{n}$, between the wave numbers obtained from the field measurements and the wave numbers corresponding to the assumed ocean model are related to $\Delta C(z)$ by the integral equation. ${ }^{1}$

$$
\Delta k_{n}=\frac{-1}{k_{b n}} \int_{0}^{\infty} \frac{\omega^{2} \Delta C(z)}{C_{b}^{3}(z) \rho(z)}\left|\phi_{b n}(z)\right|^{2} d z .
$$

In Eq. (10), which is obtained by a linearizing process, $\phi_{b n}(z)$ is the eigenfunction of the $n$th mode for the assumed ocean model, and $\rho$ is the known density of the sediment layers. By solving this integral equation, we can obtain the quantity $\Delta C(z)$ and, hence, the true compressional wave speed profile. Since the integral equation is nonlinear, the solution is obtained iteratively. This approach has been extended to include inversion for sediment density profile and the attenuation profile. ${ }^{12,13}$

Let us now consider an ocean model in which the horizontally stratified medium is represented as a set of homogeneous layers, i.e., the compressional wave speed in each layer is a constant. Let there be $M$ layers, where Layer 1 represents the water column and the remaining $M-1$ layers represent the sediment. We can then write Eq. (10) as

$$
\begin{aligned}
\Delta k_{n}= & \frac{-1}{k_{b n}}\left[\frac{\omega^{2} \Delta C_{1}}{C_{b 1}^{3} \rho_{1}} \int_{0}^{z_{1}}\left|\phi_{b n}(z)\right|^{2} d z+\cdots\right. \\
& \left.+\frac{\omega^{2} \Delta C_{M}}{C_{b M}^{3} \rho_{M}} \int_{z_{M-1}}^{z_{M}}\left|\phi_{b n}(z)\right|^{2} d z\right] .
\end{aligned}
$$

In Eq. (11), the quantities $\rho_{m}$ and $C_{b m}$ are the density and the initial estimate of the compressional wave speed in the $m$ th layer, respectively, and $\Delta C_{m}$ is the correction to the compressional wave speed in the $m$ th layer that we seek to determine. For a set of $N$ modal wave numbers, Eq. (11) can be represented by the matrix equation

$$
\Delta \mathbf{k}=\mathbf{A} \Delta \mathbf{C},
$$

where $\Delta \mathbf{k}$ is a vector containing the wave number differences for $N$ modes, $\Delta \mathbf{C}$ is a vector of length $M$ containing the corrections to the compressional wave speed for the $M$ layers, and $\mathbf{A}$ is an $N \times M$ matrix whose element $A_{n m}$ is given by the expression 


$$
A_{n m}=\frac{-1}{k_{b n}} \frac{\omega^{2}}{C_{b m}^{3} \rho_{m}} \int_{z_{m-1}}^{z_{m}}\left|\phi_{b n}(z)\right|^{2} d z .
$$

The element $A_{n m}$ represents the change in the modal wave number of the $n$th mode due to unit change in the compressional wave speed in the $m$ th layer. It is apparent from this formulation that the matrix $\mathbf{A}$ and the vector $\mathbf{\Delta C}$ can be augmented to include estimation of other parameters. For example, if we need to estimate the water column thickness, we can augment the matrix by one column with each element of this column representing the sensitivity of the corresponding modal wave number to changes in the water column thickness, and the vector $\mathbf{\Delta} \mathbf{C}$ augmented to include $\Delta h$ the correction to be applied to the water column thickness. The sensitivity of the modal wave number to changes in the water column thickness can be obtained using the expression derived in Ref. 14.

We have noted earlier that noise in the data creates noise in the wave number spectrum, which affects the values of the wave numbers of the higher-order modes. The inversion was therefore performed using the wave numbers of the dominant modes, viz. the lower-order modes, which are much less affected by noise in the data. The wave numbers of the dominant modes at $18 \mathrm{~Hz}, 20 \mathrm{~Hz}, 25 \mathrm{~Hz}$, and $30 \mathrm{~Hz}$ were used as data. The integral Eq. (10) was replaced by a set of equations, as shown below, to incorporate multifrequency inversion:

$$
\begin{aligned}
& \Delta k_{n}\left(\omega_{p}\right)=\frac{-1}{k_{b n}\left(\omega_{p}\right)} \int_{0}^{\infty} \frac{\omega_{p}^{2} \Delta C(z)}{C_{b}^{3}(z) \rho(z)}\left|\phi_{b n}\left(z, \omega_{p}\right)\right|^{2} d z ; \\
& n=1, \cdots, N, \quad p=1, \cdots, P .
\end{aligned}
$$

This set of integral equations is reduced to a matrix equation of the form

$$
\left[\begin{array}{c}
\Delta \mathbf{k}\left(\omega_{1}\right) \\
\cdot \\
\cdot \\
\Delta \mathbf{k}\left(\omega_{\mathbf{P}}\right)
\end{array}\right]=\left[\begin{array}{c}
\mathbf{A}\left(\omega_{1}\right) \\
\cdot \\
\cdot \\
\cdot \\
\mathbf{A}\left(\omega_{\mathbf{P}}\right)
\end{array}\right]\left[\begin{array}{c}
\Delta \mathbf{c}_{1} \\
\cdot \\
\cdot \\
\Delta \mathbf{c}_{\mathbf{M}}
\end{array}\right],
$$

where the left-hand side is a vector made up of $P$ subvectors $\Delta \mathbf{k}\left(\omega_{\mathbf{p}}\right)$. Each of these subvectors represents the differences in wave numbers at frequency $\omega_{p}$. On the right-hand side, the matrices $\mathbf{A}\left(\omega_{\mathbf{p}}\right)$ are the kernels of the integral equations corresponding to the different frequencies. The vector on the right-hand side is a vector of the corrections to the compressional wave speed of the layers, and this is determined by solving the matrix Eq. (15).

The following assumptions are made in performing the inversion:

1. The sediment layers are assumed to be fluid, and

2. The density and attenuation of the sediment layers are assumed to have a constant value in each sediment layer and in the termination half-space. These values are assumed to be known. The acoustic field in the water column is most sensitive to the compressional wave speed of the sediments and water column, and its sensitivity to sediment density and attenuation is small in comparison. Therefore, errors in the assumed values of density and attenuation of the sediment layers will not affect the estimates of the sediment compressional wave speed.

\section{INVERSION RESULTS}

\section{A. Estimate of compressional wave speed profiles and water column thickness}

As stated earlier, the acoustic parameters of the water column were not provided by the survey agency that collected the data. Only an approximate value of the water column thickness was provided. It was therefore necessary to consider the water column sound speed and the water column thickness as unknown parameters in the inversion process.

Initial estimates of the sound speed in the water column and the water column thickness were made assuming a Pekeris model for the ocean. Modal wave numbers of Mode one at frequencies from 80 to $100 \mathrm{~Hz}$ were obtained from Shot 50 data. The inverse problem was formulated with three unknown parameters; namely, the sound speed in the water column, the water column thickness, and the compressional wave speed in the half-space representing the sediments. At these frequencies, the influence of the mode function is mostly restricted to the water column; and this influence decays as we move away from the water sediment interface. This preliminary estimate gave the water column sound speed as $1540 \mathrm{~m} / \mathrm{s}$, the water column thickness as $36.9 \mathrm{~m}$ and the sound speed in the sediment as $1700 \mathrm{~m} / \mathrm{s}$.

The initial ocean model, therefore, consisted of a water column that was $36.9 \mathrm{~m}$ thick with the sound speed in the water column set as $1540 \mathrm{~m} / \mathrm{s}$. This is followed by three homogeneous sediment layers of thickness $35 \mathrm{~m}$, in which the compressional wave speed was set at $1700 \mathrm{~m} / \mathrm{s}$. The density and compressional wave attenuation in the sediment layers were assumed to be $1.7 \mathrm{gm} / \mathrm{cc}$ and $0.1 \mathrm{~dB} /$ acoustic wavelength. The half-space beyond the layers was assumed to have a compressional wave speed of $2100 \mathrm{~m} / \mathrm{s}$. This value was based on the phase speeds of the propagating modes at the frequencies considered for analysis. The values of density and attenuation in the half-space were also set at $1.7 \mathrm{~g} / \mathrm{cm}^{3}$ and $0.1 \mathrm{~dB} /$ acoustic wavelength.

The inversions were then carried out for the compressional wave speed in the water column and sediment layers, and for the thickness of the water column. The wave numbers of Modes 1 and 2 at frequencies of $18 \mathrm{~Hz}, 20 \mathrm{~Hz}$, and $25 \mathrm{~Hz}$, and Mode 1 only at $30 \mathrm{~Hz}$, were used as data. The thicknesses of the sediment layers were excluded from the estimation process, because the inversion process with lowfrequency data yields only the depth-averaged compressional wave speed over the assumed layer thicknesses. The sensitivity of modal wave numbers to changes in the sound speed in the water column and the sediment layers were obtained from the expressions in Eq. (13). The sensitivity of the modal wave number to changes in water column depth was obtained numerically. 

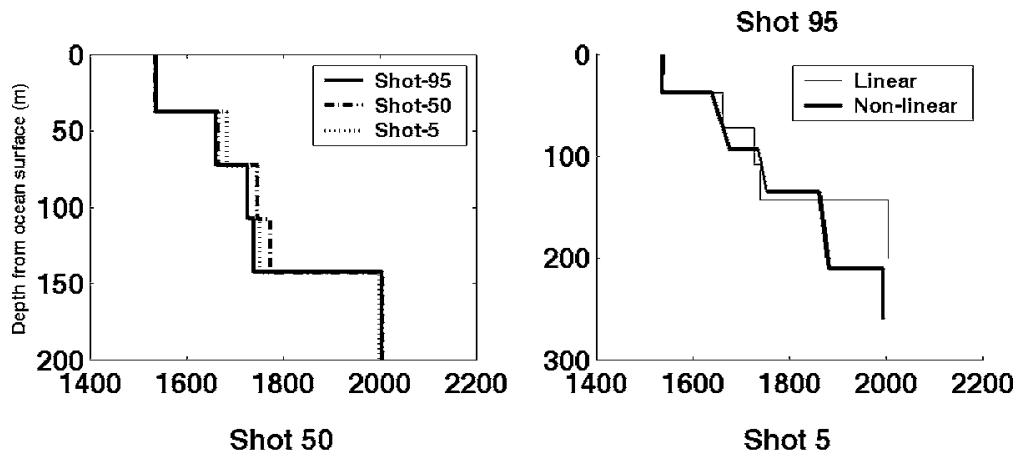

FIG. 4. The top left panel shows the compressional wave speed profiles determined from the three shot data. The remaining panels show the comparison between the profiles obtained from linear inverse method based on the modal wave numbers and the mean value
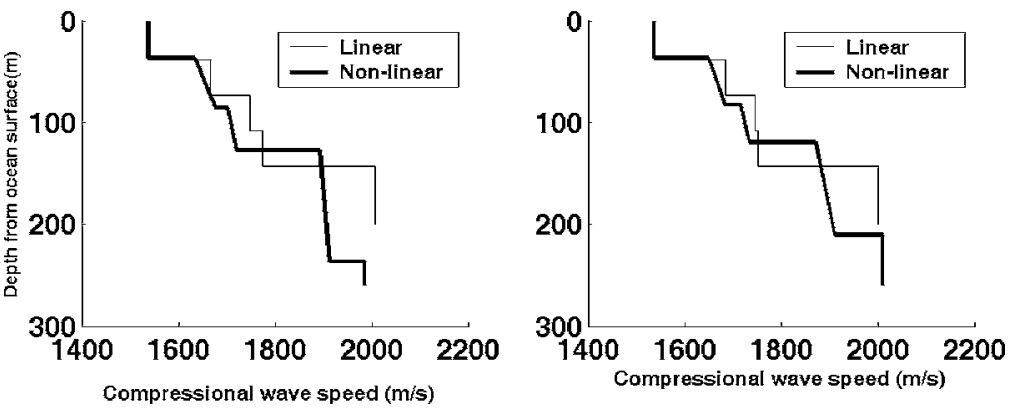
of layer speeds determined through Gibb's sampling (Ref. 2).

The models obtained by the linear inverse for the three shot data are shown in the top left panel of Fig. 4. There are no in situ measurements of sediment properties to validate the compressional wave speed models obtained from modal inverse. We, therefore, evaluate this model by comparing:

(a) The model with that obtained from a nonlinear method,

(b) The pressure field predicted by the model with the field obtained from the measured field data, and

(c) The wave number spectra from the model with the spectra obtained from the measured field data.

For comparison, the other three panels in Fig. 4 show the compressional wave speed models obtained from the nonlinear method based on matched field processing, ${ }^{2}$ together with the models from linear inverse. The values in the figure for the wave speeds, as determined by a nonlinear method, are the mean values obtained by Gibb's sampling. ${ }^{2}$ It is seen that the estimates of the water column sound speed and thickness, obtained by the linear method, agree well with the estimates from matched field processing. The compressional wave speed in the top three sediment layers follow the trend seen in the model from matched field processing. To make the comparison meaningful, the mean values and the deviation estimates from the Gibb's sampling results were depth averaged over the layer intervals in the linear model, and are presented in Table I. It can be seen in Table I that the modal inverse estimates of the compressional wave speed, in the sediment layers up to a sediment depth of $105 \mathrm{~m}$, are within two times the standard deviation from the mean values predicted by Gibb's sampling for models from Shots 50 and 95. However, in the case of Shot 5, similar agreements between the estimates exist only up to a sediment depth of $70 \mathrm{~m}$.

The fields predicted by the model from modal inverse are compared with the field obtained from the field measurement in Fig. 5. We note that the field from the measured field data has a lot of structure. We have shown, by using synthetic data (Fig. 3), that the structure in the measured field is the result of noise in the data. The model-predicted field follows the general trend of the measured field at the lower frequencies. In these cases, the fields are dominated by the lower-order modes, and the wave numbers of these modes were used in the inverse. A comparison of the wave number spectra obtained from field measurements at $20 \mathrm{~Hz}$ and the model predicted spectrum is shown in Fig. 6. These plots show good agreement with respect to Modes 1 and 2 .

Finally, the correlation between the measured field and

TABLE I. Comparison of wave speeds in the sediment layers. In the nonlinear method (Ref. 2) (matched field processing), both the thickness and the wave speeds of the sediment layers were estimated. In the linear inverse method based on modal wave numbers, the sediment layers were assumed to be $35 \mathrm{~m}$ thick and with homogeneous properties. To make the comparison meaningful, the profiles obtained by the nonlinear method were depth averaged to estimate the wave speeds in the layering adopted by the linear method.

\begin{tabular}{|c|c|c|c|c|c|c|c|c|c|}
\hline \multirow{2}{*}{$\begin{array}{l}\text { Depth into } \\
\text { sediment } \\
\quad(\mathrm{m})\end{array}$} & \multicolumn{3}{|c|}{ Shot 5} & \multicolumn{3}{|c|}{ Shot 50} & \multicolumn{3}{|c|}{ Shot 95} \\
\hline & Mean & Deviation & Model & Mean & Deviation & Model & Mean & Deviation & Model \\
\hline $0-35$ & 1664 & 13.5 & 1684 & 1656 & 13.7 & 1667 & 1658 & 14.2 & 1662 \\
\hline $35-70$ & 1720 & 25.9 & 1745 & 1705 & 24.5 & 1748 & 1694 & 20.0 & 1727 \\
\hline $70-105$ & 1836 & 23.8 & 1754 & 1804 & 26.1 & 1774 & 1779 & 27.1 & 1749 \\
\hline $105-175$ & 1890 & 19.1 & 2008 & 1892 & 18.7 & 2008 & 1898 & 18.6 & 2005 \\
\hline
\end{tabular}



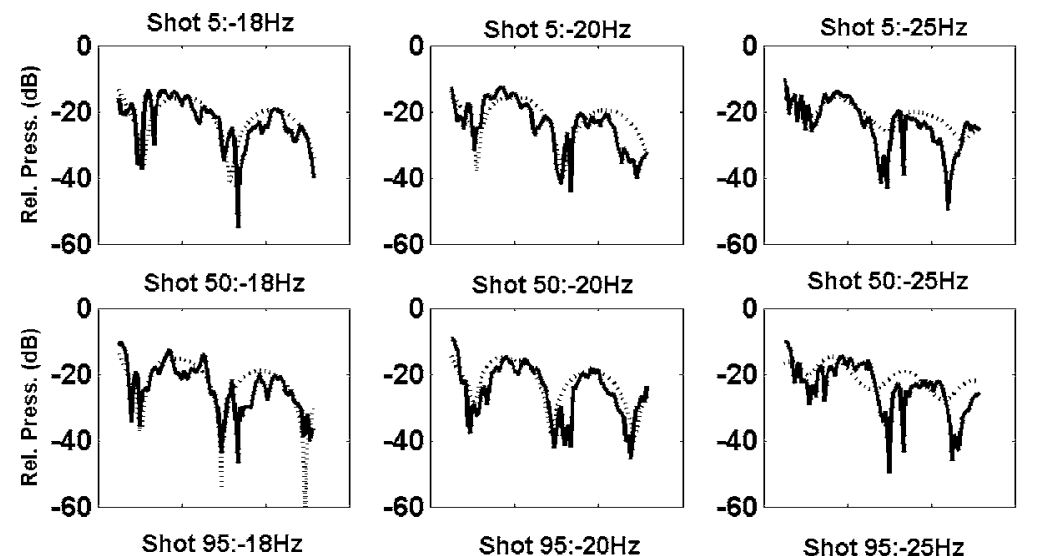

FIG. 5. The magnitude of the pressure field at three different frequencies obtained from the field data (full lines) are compared with the fields predicted by the models (dotted lines) for Shots 5, 50, and 95.
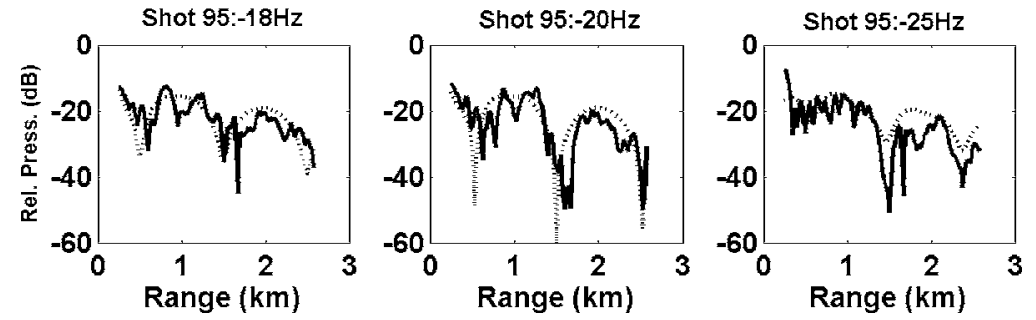

the predicted field are given in Table II. The correlation between the measured field and the predicted field is given by the expression

$$
C\left(\mathbf{P}_{\text {meas }}, \mathbf{P}_{\text {predict }}\right)=\frac{\left|\mathbf{p}_{\text {meas }}^{\mathbf{H}} \mathbf{p}_{\text {predict }}\right|}{\sqrt{\mathbf{p}_{\text {meas }}^{\mathbf{H}} \mathbf{p}_{\text {meas }}} \sqrt{\mathbf{p}_{\text {predict }}^{\mathbf{H}} \mathbf{p}_{\text {predict }}}},
$$

where $\mathbf{p}_{\text {meas }}=\left[p_{\text {meas }}(1) \cdots p_{\text {meas }}(N)\right]^{T}$ is the vector containing the measure field at $N$ receivers and $\mathbf{p}_{\text {predict }}$ $=\left[p_{\text {predict }}(1) \cdots p_{\text {predict }}(N)\right]^{T}$ is a vector containing the modelpredicted fields at the same set of receivers. The superscripts $T$ and $H$ denote transpose and conjugate transpose, respectively.

The correlation has fairly high values for lower frequencies, and reduces appreciably for $30 \mathrm{~Hz}$. As already mentioned, the data used in the inversion included the wave number of Mode 1 only at the frequency of $30 \mathrm{~Hz}$. Hence, the models obtained with these data are not likely to have adequate resolution to predict the field of $30 \mathrm{~Hz}$. This has resulted in a low correlation value at this frequency.

\section{B. Resolution and variance of estimates \\ 1. Resolution}

The linearized version of the inverse problem represented by Eq. (15) is of the form

$$
\mathbf{G m}=\mathbf{d}+\boldsymbol{\nu},
$$

where $\mathbf{G}$ is a matrix containing the kernel, $\mathbf{m}$ is the model to be estimated, $\mathbf{d}$ is the data vector, and $\boldsymbol{\nu}$ represents noise in the data. If the problem is linear, the resolution matrix $\mathbf{R}$ is given by the expression ${ }^{15}$
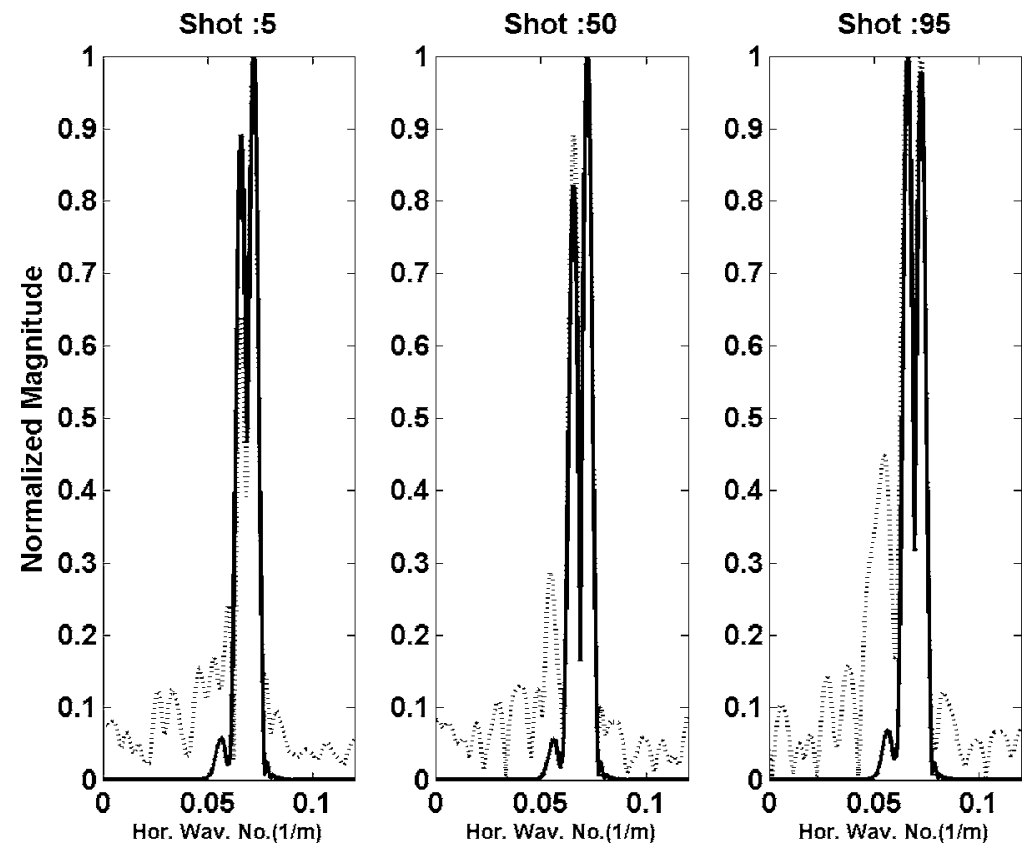

FIG. 6. The wave number spectrum obtained from field data (dotted lines) is compared with the spectrum obtained from the predicted field (full lines). The spectra from the three shots at $20 \mathrm{~Hz}$ are shown. 
TABLE II. Correlation values between the measured field and the predicted field obtained from modal inverse models.

\begin{tabular}{cccc}
\hline \hline Frequency $(\mathrm{Hz})$ & Shot 5 & Shot 50 & Shot 95 \\
\hline 18 & 0.83 & 0.66 & 0.70 \\
20 & 0.86 & 0.90 & 0.87 \\
25 & 0.83 & 0.84 & 0.73 \\
30 & 0.80 & 0.55 & 0.62 \\
\hline \hline
\end{tabular}

$$
\mathbf{R}=\left(\mathbf{G}^{\mathbf{T}} \mathbf{C}_{\nu}^{-1} \mathbf{G}+\mathbf{C}_{\mathbf{m}}^{-1}\right)^{-1}\left(\mathbf{G}^{\mathbf{T}} \mathbf{C}_{\nu}^{-1} \mathbf{G}\right) .
$$

In Eq. (18), $\mathbf{C}_{\boldsymbol{\nu}}$ and $\mathbf{C}_{\mathbf{m}}$ are the data and model covariance matrices, respectively. When the nonlinear inverse problem is solved iteratively as a sequence of linear inverse problems, the matrix $\mathbf{G}$ in Eq. (18) is replaced by a matrix that uses the final solution to determine the elements of the resolution matrix $\mathbf{R}$.

The data errors were assumed to be uncorrelated, yielding a diagonal matrix for the data covariance matrix. The error $\varepsilon$ in the estimation of the modal wave numbers is related to the aperture of the horizontal array and the signal to noise ratio (SNR), and is given by the $\operatorname{expression}^{1} \varepsilon$ $=2 \pi / L \sqrt{\mathrm{SNR}}$, where SNR is the signal to noise ratio and $L$ is the length of the horizontal array over which the acoustic field is measured. The data errors were obtained using a SNR based on the average value of the signal to noise of the modal peaks, as determined from the wave number spectrum. The model covariance matrix was also assumed to be diagonal with the standard deviation of the error in the compressional wave speed of the sediment layers estimated as $20 \mathrm{~m} / \mathrm{s}$, and that of the sound speed in the water column assumed to be $5 \mathrm{~m} / \mathrm{s}$. The estimate of model variance for the sediment layers is based on the compressional wave speed variability for silty sediments. ${ }^{16}$ A much smaller variability has been assumed for the sound speed in the water column.

The resolution matrix determines how well the estimated parameters are resolved. If they are well resolved, then the resolution matrix will be a diagonal matrix. Its deviation from the ideal is determined by the resolution length which is given by the expression

$$
r l(i)=\frac{\sum_{j=1}^{M} R_{i j}^{2} d z}{R_{i i}^{2}},
$$

where $\operatorname{rl}(i)$ is the resolution length of the $i$ th layer, $d z$ is the layer thickness, and $R_{i j}$ is the element in the $i$ th row and $j$ th column of the $\mathbf{R}$ matrix. The resolution length is a measure of the interval over which the estimate is averaged. It is seen from Eq. (19) that if $\mathbf{R}$ is a diagonal matrix, the resolution length of each layer is equal to the layer thickness.

The left panels of Fig. 7 show the resolution length of each layer for one of the three shots. The plot shows the resolution length for six layers. In our inverse, we obtained the wave speed for the water column and three sediment layers. Layers 5 and 6 represent the half-space. It is seen that for the top four layers the resolution lengths are approximately $47 \mathrm{~m}$. The subsequent resolution length for the halfspace is much higher. It is to be noted that only lower-order modes were used in the inversion; hence, the poor resolution at greater depths.

\section{Variance}

For the linear problem of Eq. (17), the variance of the solution can be determined from the expression ${ }^{16}$

$$
\hat{\mathbf{C}}_{\mathbf{m}}=\left(\mathbf{G}^{\mathbf{T}} \mathbf{C}_{\nu}^{-1} \mathbf{G}+\mathbf{C}_{\mathbf{m}}^{-1}\right)^{-1},
$$

where $\hat{\mathbf{C}}_{\mathbf{m}}$ is the a posteriori covariance matrix of the model parameters. The diagonal of this matrix shows the variance of the model parameters. One would expect the variance of model parameters to reduce after inversion. If the variance
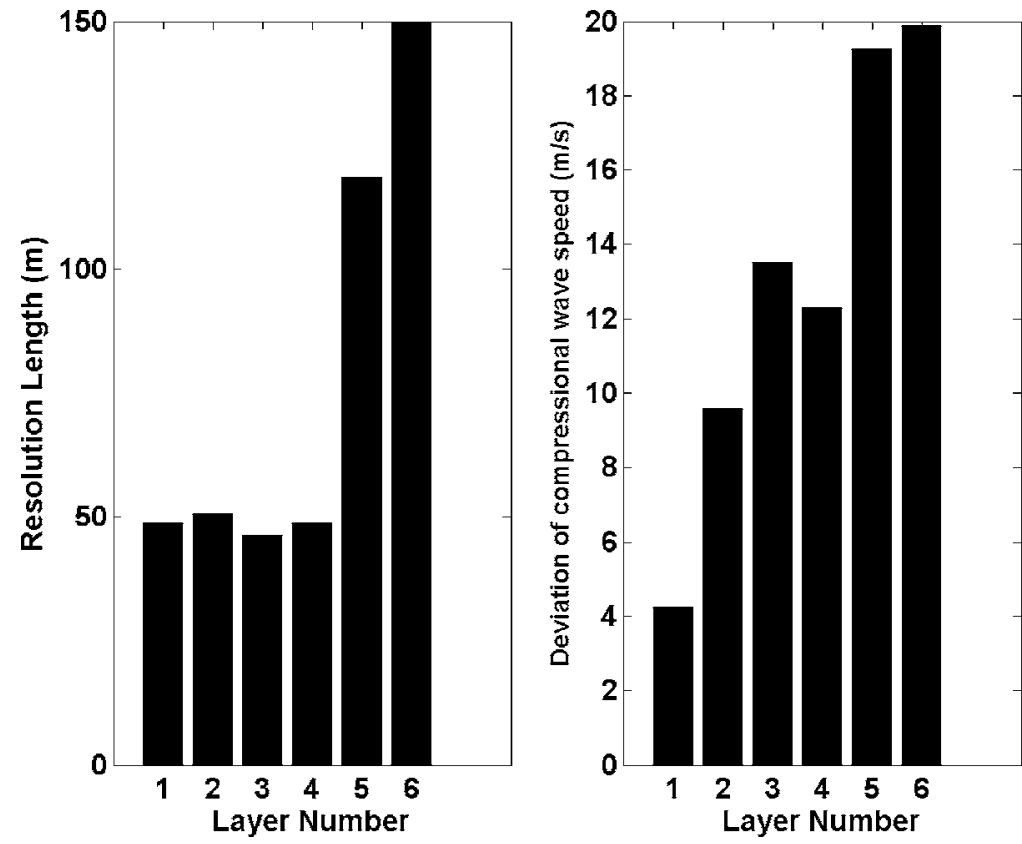

FIG. 7. The panels on the left show the resolution lengths at different layers for one of the three shots. Layer 1 is the water column. Layers 2 to 4 correspond to three sediment layers. Layers 5 and 6 represent the sediment in the half-space. The water column is approximately $37 \mathrm{~m}$ thick, while the sediment layers are modeled as $35 \mathrm{~m}$ thick. The right panel shows the deviation of the compressional wave speed estimate in each layer. 
does not change after inversion, it implies that the inverse has little impact on these model parameters.

The posteriori model standard deviation determined from Eq. (20) is shown in the right panels of Fig. 7. It is seen that the posteriori value is less than the prior value in Layers 1 to 4 . For Layers 5 and 6, there is very little change in the standard deviation. This indicates that the inverse did not have any effect on the deeper layers, a conclusion that is supported by the resolution estimates as well.

\section{CONCLUSIONS}

The data used in this analysis were broadband signals acquired on a horizontal array during a geophysical survey. Such data are routinely collected by oil companies, and an important thrust of this research is to determine whether such data can be used to determine near-surface geoacoustic properties of the sediment. This paper looks at estimating the sediment acoustic properties from estimates of modal eigenvalues obtained from the broadband data. Sources of errors in the estimation of the modal eigenvalues were considered. Since the water column properties were not known, joint inversion of the water column and sediment acoustic properties was carried out.

The source was an air gun with most of its energy in the frequency band of $15-50 \mathrm{~Hz}$. The receiver array consisted of 96 hydrophones with an interelement spacing of $25 \mathrm{~m}$. The data were transformed into the frequency domain, and thus the pressure field, as a function of range at a given frequency, was extracted from the data. We have used these data to extract the horizontal wave numbers of the propagating modes, and attempted to obtain a water column and sediment model using these wave numbers.

The pressure field data obtained from the horizontal array have a spatial sampling of $25 \mathrm{~m}$. At frequencies higher than $30 \mathrm{~Hz}$, the spatial sampling rate is below the Nyquist rate required to avoid aliasing when transforming the pressure field into the wave number spectrum. We demonstrated with synthetic data that interpolation of the data at the required sampling rate does not introduce errors in the estimate of modal wave numbers. We also investigated the effect of errors in the assumed range and receiver depth. Our simulations indicated that the effect of these errors on modal wave number estimates of the dominant low-order modes is negligible. The addition of random Gaussian noise to the simulated broadband data results in considerable changes in both the pressure field obtained from it and in the corresponding wave number spectrum. But the dominant modes seem to be unaffected by noise. The weaker higher-order modes are affected, and spurious modes appear at wave numbers higher than the water wave number. This results in a highly oscillatory pressure-field structure. It is to be noted that the pressure fields obtained from the field experiment also exhibited a similar structure. It was therefore decided to use the wave numbers of dominant modes (Modes 1 and 2) only for the parameter estimation. The wave numbers of dominant modes at $18,20,25$, and $30 \mathrm{~Hz}$ were used, and the joint water column-sediment bottom model was determined from Shot 5, Shot 50, and Shot 95 data.
The estimates of water column sound speed and water column thickness are very close to the values obtained through matched field processing. The estimates of sediment compressional wave speed up, to a sediment depth of $105 \mathrm{~m}$, are within two standard deviations of the mean values calculated using Gibb's sampling approach ${ }^{2}$ in the case of models from Shot 50 and Shot 95 data. In the case of Shot 5, the agreement is limited to sediment depth less than $70 \mathrm{~m}$. The penetration of these modes is restricted to the top layers; hence, the poor resolution for the deeper layers.

The comparison between the fields predicted by the models and the measured fields indicates good correlation at the lower frequencies. At $30 \mathrm{~Hz}$, the correlation is poor because only the wave number of Mode 1 at this frequency was incorporated in the data.

The resolution estimates indicate that the top layers are fairly well resolved, and this conclusion is supported by the variance estimates.

Errors in the data impose restrictions on the modal wave numbers that can be used as data in the linear inverse procedure. This results in poor resolution of the deeper layers. However, the estimates of the shallower layers are comparable to the values obtained by matched field processing. This suggests that the modal inverse method can be used to determine the initial model and the search range for a global optimization procedure, thus providing improved efficiency in the execution of matched field processing.

\section{ACKNOWLEDGMENTS}

The work was supported by the Naval Research Board, Ministry of Defense, Government of India.

${ }^{1}$ S. D. Rajan, J. F. Lynch, and G. V. Frisk, "Perturbative inverse methods for obtaining bottom geoacoustic parameters in shallow water," J. Acoust. Soc. Am. 82, 998-1017 (1987).

${ }^{2}$ P. V. Nagesh, S. D. Rajan, and G. V. Anand, "Estimation of sediment acoustic properties from horizontal array data," Conf. on Underwater Acoustic Measurements: Technologies and Results, Heraklion, Crete, Greece, (2005).

${ }^{3}$ J. F. Lynch, S. D. Rajan, and G. V. Frisk, "A comparison of broadband and narrowband inversions for bottom geoacoustic properties at a site near Corpus Christi, Texas," J. Acoust. Soc. Am. 89, 648-651 (1991).

${ }^{4}$ G. V. Frisk, J. F. Lynch, and S. D. Rajan, "Determination of wave speed profiles using modal inverse techniques in a range-dependent environment in Nantucket Sound," J. Acoust. Soc. Am. 86, 1928-1939 (1989).

${ }^{5}$ G. V. Frisk and J. F. Lynch, "Shallow water characterization using Hankel transform," J. Acoust. Soc. Am. 76, 205-211 (1980).

${ }^{6} \mathrm{H}$. Schmidt, Oases version 2.2, User Guide and Reference Manual (Massachusetts Institute of Technology, Cambridge, MA, 1999).

${ }^{7}$ F. B. Jensen, W. A. Kuperman, M. B. Porter, and H. Schmidt, Computational Ocean acoustics (Springer, New York, 2000).

${ }^{8}$ K. E. Hawker, "A normal mode theory of acoustic Doppler effects in the ocean waveguides," J. Acoust. Soc. Am. 56, 58-64 (1974).

${ }^{9}$ H. Schmidt and W. A. Kuperman, "Spectral and modal representations of the Doppler-shifted field in ocean waveguides," J. Acoust. Soc. Am., 96, 386-395 (1994).

${ }^{10}$ K. M. Becker, S. D. Rajan, and G. V. Frisk, "Results from geoacoustic inversion techniques workshop using modal inverse method," IEEE J. Ocean. Eng. 28, 331-341 (2003).

${ }^{11}$ S. D. Rajan and S. Bhatta, "Evaluation of high-resolution methods for estimating modal modal wave numbers in a shallow water environment," J. Acoust. Soc. Am. 93, 378-389 (1993). 
${ }^{12}$ S. D. Rajan, "Simultaneous reconstruction of compressional wave speed and density profiles from modal eigenvalues," J. Comput. Acoust. 6, 257268 (1998).

${ }^{13}$ S. D. Rajan, G. V. Frisk, and J. F. Lynch, "On the determination of modal attenuation coefficients and compressional wave attenuation profiles in a range-dependent environment in Nantucket sound," IEEE J. Ocean. Eng. 17, 118-126 (1992).
${ }^{14}$ R. T. Kessel, "The variation of modal wave numbers with geoacoustic parameters of a layered media," J. Acoust. Soc. Am. 102, 2690-2696 (1997).

${ }^{15}$ S. D. Rajan, "Waveform inversion to obtain the geoacoustic properties of sediments in deep water," J. Acoust. Soc. Am. 91, 3228-3241 (1992).

${ }^{16}$ E. L. Hamilton, "Geoacoustic modeling of the seafloor," J. Acoust. Soc. Am. 68, 1313-1340 (1980). 